\title{
New Solanocapsine-Type Tomato Glycoside from Ripe Fruit of Solanum lycopersicum
}

\author{
Mizuho OhNo, ${ }^{a}$ Masateru Ono, ${ }^{b}$ and Toshihiro NoharA ${ }^{*, a}$ \\ ${ }^{a}$ Faculty of Pharmaceutical Sciences, Sojo University; 4-22-1 Ikeda, Kumamoto 860-0082, Japan: and ${ }^{b}$ School of \\ Agriculture, Tokai University; 5435 Aso, Kumamoto 869-1404, Japan. \\ Received May 26, 2011; accepted August 18, 2011; published online August 23, 2011
}

A new solanocapsine-type tomato glycoside, a novel and interesting natural steroidal glycoside, was isolated from a mini tomato, Solanum lycopersicum $L$. The chemical structure of the new minor glycoside, esculeoside B-5 (3), was determined to be $(5 S, 22 R, 23 S, 24 R, 25 S)-22,26$-epimino-16 $\beta, 23$-epoxy-3 $\beta, 23,24$-trihydroxycholestane 3-O- $\beta$-lycotetraoside.

Key words tomato fruit; Solanum lycopersicum; solanocapsine-type glycoside; tomato glycoside

For metabolic analysis of steroidal glycosides, ${ }^{1}$ we selected the tomato as part of our systematic investigation of the constituents of Solanum plants. ${ }^{2)}$ We recognized that the tomato would be appropriate because we were convinced that it had steroidal glycosides, as its aerial parts and immature fruits are rich sources of tomatine. Hence, we isolated steroidal glycosides from the ripe fruit. In Japanese tomatoes, mini tomatoes, Momotaro tomatoes, and midi tomatoes, we were the first to identify a steroidal saponin named esculeoside A (1) as a major tomato saponin in a yield of 0.0015 $0.046 \% .^{3-5)}$ From Italian tomatoes, we isolated esculeoside B-1 (2) in a yield of $0.019 \%{ }^{3,4)}$

As other minor constituents of the ripe fruits of mini, midi, and Momotaro tomatoes, we have isolated many steroidal compounds so far by using various column chromatographies on Diaion HP-20, Chromatorex NH, and silica gel, as well as high-performance liquid chromatography on octadecylsilyl (ODS) silica, as shown in Fig. 1..$^{6-8)}$

Esculeoside B-1 (2) corresponds to an isomer of esculeoside A (1) $\left.{ }^{9}{ }^{9}\right)$ Detailed investigation of the constituents of local Italian tomatoes, processed tomato products, and imported canned tomatoes, tomato juice, and ketchup is currently in progress.

Esculeoside B-1 (2) is the first example of a solanocapsine-type steroidal alkaloid glycoside, the fundamental skeleton of which is very rare and interesting in terms of its chemical structure and unknown pharmacological activity.

Here, we report one example of its rare solanocapsine-type glycoside, esculeoside B-5 (3). A commercial mini tomato was blended briefly with water by a mixer and filtered with a filter paper; the resulting filtrate was then passed through a highly porous polystyrene gel (Diaion HP-20) after elution with water. The water eluate was discarded, and further elution with $\mathrm{MeOH}$ was performed to produce an eluate that was evaporated to produce a residue. The $\mathrm{MeOH}$ eluate was also subjected to reversed-phase silica gel column chromatography on ODS and elution with $60 \% \mathrm{MeOH}$, of which the eluate was evaporated to give a residue. It consisted almost entirely of esculeoside $\mathrm{A}$; it was further chromatographed on silica gel with $\mathrm{CHCl}_{3}: \mathrm{MeOH}: \mathrm{H}_{2} \mathrm{O}=7: 3: 0.5$ to

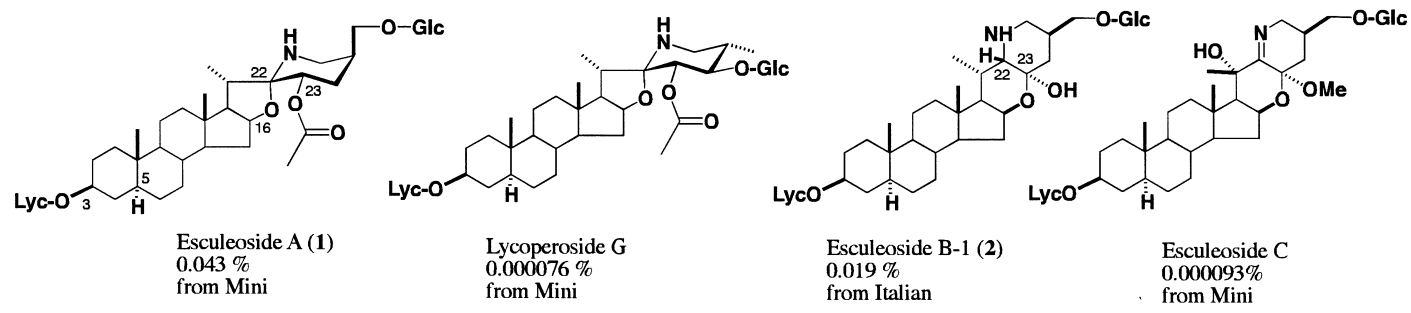

16-Acyl-pregnane-Type

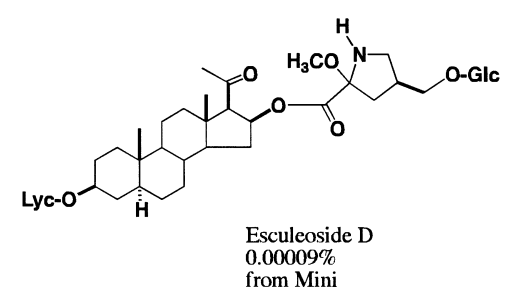

16,22-Diketone-Type

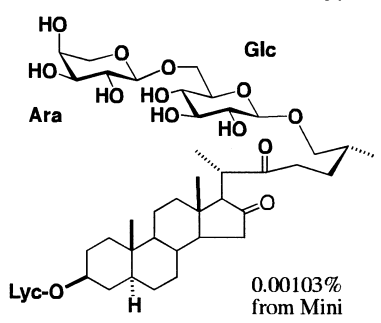

$\gamma$-Lactone-Type

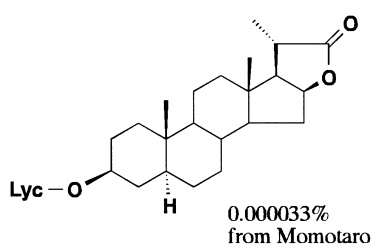

Fig. 1. Steroidal Constituents in Ripe Fruits of Tomato Obtained in Our Group 
produce five fractions. Fraction 4 was was further rechromatographed on silica gel with $\mathrm{CHCl}_{3}: \mathrm{MeOH}: \mathrm{H}_{2} \mathrm{O}=6: 4: 1$ to provide a steroidal glycoside, esculeoside B-5 (3).

Esculeoside B-5 (3) was obtained as an amorphous powder having $[\alpha]_{\mathrm{D}}-52.5^{\circ}$ (pyridine). Positive fast atom bombardment mass spectroscopy (FAB-MS) revealed a quasimolecular ion peak at $m / z 1130.5357$ due to $\left[\mathrm{C}_{52} \mathrm{H}_{85} \mathrm{NO}_{24} \mathrm{Na}\right.$ : $1130.5359]^{+}$. The ${ }^{1} \mathrm{H}-\mathrm{NMR}$ (in pyridine- $d_{5}$ ) spectrum of $\mathbf{3}$ indicated two tertiary methyl groups at $\delta 0.62(3 \mathrm{H}, \mathrm{s})$ and $0.68(3 \mathrm{H}, \mathrm{s})$ and two secondary methyl groups at $\delta 1.16(3 \mathrm{H}$, $\mathrm{d}, J=6.9 \mathrm{~Hz})$ and $1.58(3 \mathrm{H}, \mathrm{d}, J=7.3 \mathrm{~Hz})$, which are characteristic of a typical steroidal sapogenol. We also observed one acetyl signal at $\delta 2.13(3 \mathrm{H}, \mathrm{s})$; two nitrogen-bearing methylene protons at $\delta 2.98(1 \mathrm{H}, \mathrm{dd}, J=2.7,10.4 \mathrm{~Hz})$ and $3.04(1 \mathrm{H}$, t-like, $J=10.4 \mathrm{~Hz})$; one acetoxyl-bearing methine proton at $\delta 5.66(1 \mathrm{H}, \mathrm{d}, J=7.8 \mathrm{~Hz})$; and four anomeric proton signals at $\delta 4.90(1 \mathrm{H}, \mathrm{d}, J=7.2 \mathrm{~Hz}), 5.20(1 \mathrm{H}, \mathrm{d}, J=7.9 \mathrm{~Hz})$, $5.25(1 \mathrm{H}, \mathrm{d}, J=8.0 \mathrm{~Hz})$, and $5.49(1 \mathrm{H}, \mathrm{d}, J=7.3 \mathrm{~Hz})$. The ${ }^{13} \mathrm{C}-$ NMR (in pyridine- $d_{5}$ ) spectrum showed signals due to a $\beta$-lycotetraosyl moiety at $\delta 103.6$ (gal C-1), 74.3 (gal C-2), 76.2 (gal C-3), 82.5 (gal C-4), 77.6 (gal C-5), 61.8 (gal C-6), 106.3 (inner glc C-1), 81.0 (inner glc C-2), 88.6 (inner glc C3), 76.9 (inner glc C-4), 79.8 (inner glc C-5), 63.7 (inner glc C-6), 106.1 (term. glc C-1), 76.8 (term. glc C-2), 79.8 (term. glc C-3), 72.3 (term. glc C-4), 78.8 (term. glc C-5), 64.1 (term. glc C-6), 106.0 (xyl C-1), 76.5 (xyl C-2), 78.6 (xyl C3), 71.8 (xyl C-4), and 68.5 (xyl C-5). When these signals were subtracted, 29 signals remained. They consisted of four methyl carbons at $\delta 13.4,15.1,18.8$, and 24.9; nine methylene carbons at $\delta 22.0,30.1,30.7,33.4,35.2,35.8,38.4,39.2$, and 41.1; six methine carbons at $\delta 36.2,36.2,46.0,56.1$,
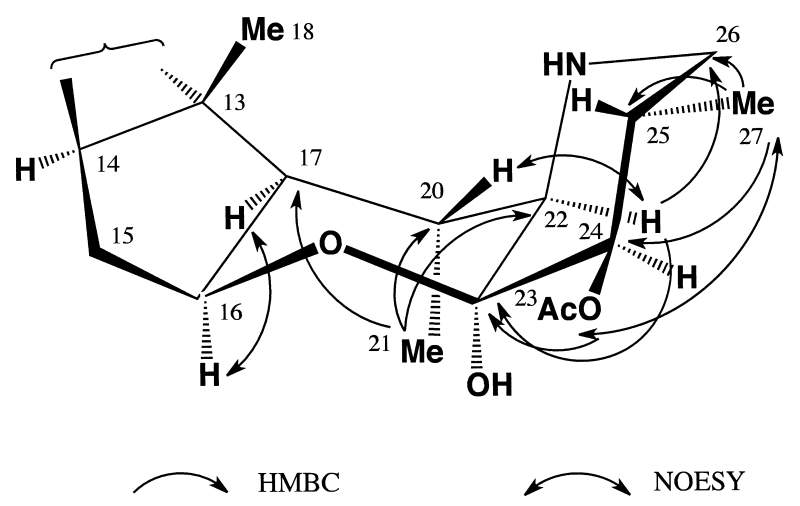

Fig. 2. Key HMBC and NOESY around D, E, F-Rings

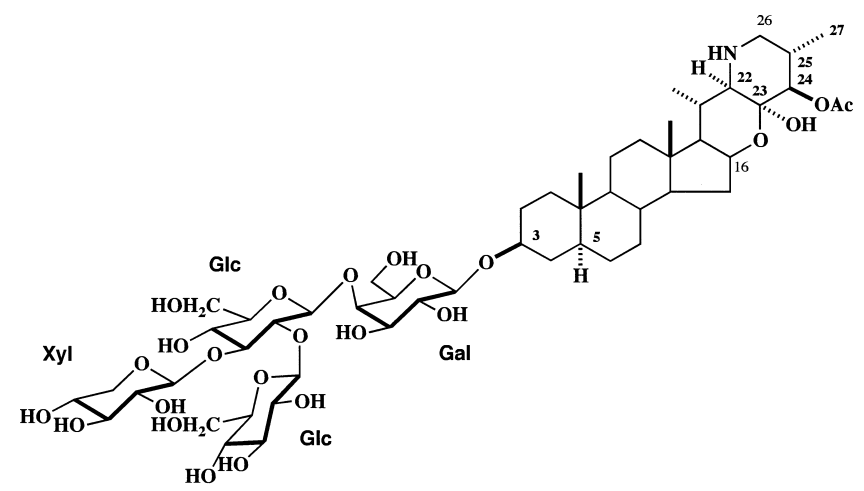

Fig. 3. Structure of Compound 3
55.0, and 31.1; two quaternary carbons at $\delta 37.0$ and 44.1; four oxygen-bearing methine carbons at $\delta 71.6,78.9$, 97.6, and 88.0; one nitrogen-bearing methine carbon at $\delta 59.7$; one acetal carbon at $\delta$ 97.6; and one acetyl group at $\delta 18.2$ and 169.2. The heteronuclear multiple bond coherence (HMBC) correlations around the steroidal $\mathrm{D}, \mathrm{E}$, and $\mathrm{F}$ rings are from $\mathrm{H}_{3}-21$ at $\delta 1.58$ to $\mathrm{C}-17$ at $\delta 59.7, \mathrm{C}-20$ at $\delta 31.1$, and $\mathrm{C}-22$ at $\delta$ 59.7; from $\mathrm{H}_{3}-27$ at $\delta 1.16$ to $\mathrm{C}-25$ at $\delta 36.2, \mathrm{C}-26$ at $\delta$ 41.1 , and $\mathrm{C}-24$ at $\delta 88.0$; from $\mathrm{H}-24$ at $\delta 5.66$ to $\mathrm{C}-23$ at $\delta$ 97.6; and from $\mathrm{H}-22$ at $\delta 3.57(1 \mathrm{H}, \mathrm{d}, J=7.8 \mathrm{~Hz})$ to $\mathrm{C}-23$ and C-26. These results revealed that esculeoside B-5 (3) has the fundamental skeleton of a solanocapsine-type glycoside, as shown in Fig. 2. Moreover, nuclear Overhauser enhancement was observed between $\mathrm{H}-20$ at $\delta 3.60$ (m) and H-22 at $\delta$ 3.57 , and between $\mathrm{H}_{3}-27$ at $\delta 1.16$ and OAc at $\delta 2.13$. Since the proton signal of $\mathrm{H}_{3}-21$ is shifted down slightly to $\delta 1.58$ because of the $23-\mathrm{OH}$ group, they lie in a 1,3-diaxial orientation. Therefore, the structure of esculeoside B-5 (3) is represented as $(5 S, 22 R, 23 S, 24 R, 25 S)$-22,26-epimino-16 $\beta, 23$ epoxy-3 $\beta, 23,24$-trihydroxycholestane $3-O-\beta$-lycotetraoside, as shown in Fig. 3. This compound might be biosynthesized from lycoperoside G. ${ }^{9}$

Solanocapsine-type glycosides such as esculeoside B-5 (3) are novel and interestingvery natural products.

\section{Experimental}

General Procedure Optical rotations were measured with a JASCO P$1020(l=0.5)$ automatic digital polarimeter. FAB-MS were obtained with a glycerol matrix in the positive ion mode using a JEOL JMS-DX300 and a JMS-DX $303 \mathrm{HF}$ spectrometer. The ${ }^{1} \mathrm{H}$ - and ${ }^{13} \mathrm{C}-\mathrm{NMR}$ spectra were measured in pyridine- $d_{5}$ with JEOL $\alpha-500$ spectrometer, and chemical shifts are given on a $\delta(\mathrm{ppm})$ scale with tetramethylsilane (TMS) as the internal standard. Column chromatographies were carried out on a Diaion HP-20 (Mitsubishi Chemical Ind., Japan), and silica gel 60 (230 - 400 mesh, Merck, Germany). TLC was performed on silica gel plates (Kieselgel $60 \mathrm{~F}_{254}$, Merck) and $\mathrm{RP} \mathrm{C}_{18}$ silica gel plates (Merck). The spots on TLC were visualized by UV light $(254 / 366 \mathrm{~nm})$ and sprayed with $10 \% \mathrm{H}_{2} \mathrm{SO}_{4}$, followed by heating.

Extraction and Isolation of Compound 3 Commercial mini tomato $(783 \mathrm{~g})$ was blended with water using a mixer for a short time $(10-20 \mathrm{~s})$ and filtered using filter paper to obtain a filtrate. The filtrate was then passed through a highly porous polystyrene gel (Diaion HP-20) and first eluted with water. The water eluate was discarded, and elution was then carried out using $\mathrm{MeOH}$ to obtain an eluate. This eluate was evaporated to obtain a residue $(23.4 \mathrm{~g})$, which was subjected to reversed-phase silica gel column chromatography, ODS, eluting with $60 \% \mathrm{MeOH}$, the eluate of which was evaporated to obtain the residue $(6.8 \mathrm{~g})$. That residue was then chromatographed on silica gel with $\mathrm{CHCl}_{3}: \mathrm{MeOH}: \mathrm{H}_{2} \mathrm{O}=7: 3: 0.5$ to obtain five fractions. Fraction 2 was almost composed of esculeoside A $(320 \mathrm{mg})$. Furthermore, fraction 4 was rechromatographed on silica gel with $\mathrm{CHCl}_{3}-$ $\mathrm{MeOH}-\mathrm{H}_{2} \mathrm{O}=6: 4: 1$ to provide a steroidal glycoside, esculeoside B-5 (3, $12 \mathrm{mg})$.

Esculeoside B-5 (3) An amorph. powder, $[\alpha]_{\mathrm{D}}-52.5^{\circ}(c=0.5$, pyridine).

Positive high resolution (HR)-FAB-MS ( $\mathrm{m} / \mathrm{z}$ ): 1130.5357 (Calcd for $\mathrm{C}_{52} \mathrm{H}_{85} \mathrm{NO}_{24} \mathrm{Na}$ : 1130.5359)

${ }^{1} \mathrm{H}-\mathrm{NMR}$ (pyridine- $d_{5}$ ) $\delta: 0.62\left(3 \mathrm{H}, \mathrm{s}, \mathrm{H}_{3}-19\right), 0.68\left(3 \mathrm{H}, \mathrm{s}, \mathrm{H}_{3}-18\right), 1.16$ $\left(3 \mathrm{H}, \mathrm{d}, J=6.9 \mathrm{~Hz}, \mathrm{H}_{3}-27\right), 1.58\left(1 \mathrm{H}, \mathrm{d}, J=7.3 \mathrm{~Hz}, \mathrm{H}_{3}-21\right), 2.13(3 \mathrm{H}, \mathrm{s}, \mathrm{OAc})$, $2.98(1 \mathrm{H}, \mathrm{dd}, J=2.7,10.4 \mathrm{~Hz}, \mathrm{Hb}-26), 3.04(1 \mathrm{H}, \mathrm{t}$-like, $J=10.4 \mathrm{~Hz}, \mathrm{Ha}-26)$, $3.57(1 \mathrm{H}, \mathrm{d}, \mathrm{J}=7.8 \mathrm{~Hz}), 4.90(1 \mathrm{H}, \mathrm{d}, J=7.2 \mathrm{~Hz}$, gal H-1), $5.20(1 \mathrm{H}, \mathrm{d}$, $J=7.9 \mathrm{~Hz}$, inner glc $\mathrm{H}-1), 5.25(1 \mathrm{H}, \mathrm{d}, J=8.0 \mathrm{~Hz}$, xyl H-1), $5.49(1 \mathrm{H}, \mathrm{d}$, $J=7.3 \mathrm{~Hz}$, term. glc $\mathrm{H}-1), 5.66(1 \mathrm{H}, \mathrm{d}, J=7.8 \mathrm{~Hz}, \mathrm{H}-24)$.

${ }^{13} \mathrm{C}-\mathrm{NMR}$ (pyridine- $d_{5}$ ) $\delta: 39.2(\mathrm{C}-1), 30.1(\mathrm{C}-2), 78.9(\mathrm{C}-3), 35.2(\mathrm{C}-4)$, 46.0 (C-5), 30.7 (C-6), 35.8 (C-7), 36.2 (C-8), 56.1 (C-9), 37.0 (C-10), 22.0 (C-11), 38.4 (C-12), 44.1 (C-13), 55.0 (C-14), 33.4 (C-15), 71.6 (C-16), 59.7 (C-17), 15.1 (C-18), 13.4 (C-19), 31.1 (C-20), 18.8 (C-21), 59.7 (C-22), 97.6 (C-23), 88.0 (C-24), 36.2 (C-25), 41.1 (C-26), 24.9 (C-27), 18.2, 169.2 (acetyl group), 103.6 (gal C-1), 74.3 (gal C-2), 76.2 (gal C-3), 82.5 (gal C4), 77.6 (gal C-5), 61.8 (gal C-6), 106.3 (inner glc C-1), 81.0 (inner glc C-2), 
88.6 (inner glc C-3), 76.9 (inner glc C-4), 79.8 (inner glc C-5), 63.7 (inner glc C-6), 106.1 (term. glc C-1), 76.8 (term. glc C-2), 79.8 (term. glc C-3), 72.3 (term. glc C-4), 78.8 (term. glc C-5), 64.1 (term. glc C-6), 106.0 (xyl C-1), 76.5 (xyl C-2), 78.6 (xyl C-3), 71.8 (xyl C-4), 68.5 (xyl C-5).

Sugar Analysis A solution of each compound (3) $(3.0 \mathrm{mg})$ in $2 \mathrm{M}$ $\mathrm{HCl}$ : dioxane $(1: 1,2 \mathrm{ml})$ was heated at $100^{\circ} \mathrm{C}$ for $1 \mathrm{~h}$. The reaction mixture was diluted with $\mathrm{H}_{2} \mathrm{O}$ and evaporated to remove dioxane. The solution was neutralized with Amberlite MB-3 and passed through a SEP-PAK $\mathrm{C}_{18}$ cartridge to give a sugar fraction. The sugar fraction was concentrated to dryness in vacuo to give a residue, which was dissolved in $\mathrm{CH}_{3} \mathrm{CN}: \mathrm{H}_{2} \mathrm{O}(3: 1$, $250 \mu \mathrm{l})$. The sugar fraction was analyzed by HPLC under the following conditions: column, Shodex RS-Pac DC-613 $(6.0 \mathrm{~mm}$ i.d. $\times 150 \mathrm{~mm}$, ShowaDenko, Tokyo, Japan); solvent, $\mathrm{CH}_{3} \mathrm{CN}: \mathrm{H}_{2} \mathrm{O}$ (3:1); flow rate; $1.0 \mathrm{ml} / \mathrm{min}$; column temperature, $70^{\circ} \mathrm{C}$; detection, refractive index (RI) and optical rotation (OR). The $t_{\mathrm{R}}(\mathrm{min})$ of sugars were as follow: D-xylose $4.2(+)$, D-galactose $7.0(+)$, D-glucose $7.2(+)$, [reference: D-xylose 4.2 (positive optical rotation: + ), D-galactose 7.0 (positive optical rotation: + ), D-glucose 7.2 (positive optical rotation: + )].

\section{References}

1) Noguchi E., Fujiwara Y., Matsushita S., Ikeda T., Ono M., Nohara T., Chem. Pharm. Bull., 54, 1312-1314 (2006).

2) Nohara T., Ikeda T., Fujiwara Y., Matsushita S., Noguchi E., Yoshimitsu H., Ono M., J. Nat. Med., 61, 1-13 (2007).

3) Fujiwara Y., Takai A., Uehara Y., Ikeda T., Okawa M., Yamauchi K., Ono M., Yoshimitsu H., Nohara T., Tetrahedron, 60, 4915-4920 (2004).

4) Nohara T., Ono M., Ikeda T., Fujiwara Y., El-Aasr M., J. Nat. Prod., 73, 1734-1741 (2010).

5) Manabe H., Murakami Y., El-Aasr M., Ikeda T., Fujiwara Y., Ono M., Nohara T., J. Nat. Med., 65, 176-179 (2011).

6) Ono M., Takara Y., Egami M., Uranaka K., Yoshimitsu H., Matsushita S., Fujiwara Y., Ikeda T., Nohara T., Chem. Pharm. Bull., 54, 237239 (2006).

7) Ono M., Shiono Y., Yanai Y., Fujiwara Y., Ikeda T. T. Nohara T., Chem. Pharm. Bull., 56, 1499-1501 (2008).

8) Yahara S., Uda N., Yoshio E., Yae E., J. Nat. Prod., 67, 500-502 (2004).

9) Yoshizaki M., Matsushita S., Fujiwara Y., Ikeda T., Ono M., Nohara T., Chem. Pharm. Bull., 53, 839—840 (2005). 